\title{
Risk factors of pulmonary tuberculosis: A community-based case control study
}

\section{Prashant Ramdas Kokiwar}

Professor and Head, Department of Community Medicine, Malla Reddy Institute of Medical Sciences, Suraram, Hyderabad, Telangana, India - 500055

\begin{tabular}{|c|c|c|c|c|c|c|c|c|}
\hline Abstract & Introduction & Methodology & Results & Conclusion & References & Citation & & les/Figures \\
\hline \multicolumn{9}{|c|}{ Corresponding Author } \\
\hline \multicolumn{8}{|c|}{$\begin{array}{l}\text { Dr Prashant Ramdas Kokiwar, Professor and Head, Department of Community Medicine, Malla Reddy } \\
\text { Institute of Medical Sciences, Suraram, Hyderabad, Telangana, India - } 500055 \\
\text { E Mail ID: pkokiwar@yahoo.com }\end{array}$} & 回部回 \\
\hline
\end{tabular}

\section{Citation}

Kokiwar PR. Risk factors of pulmonary tuberculosis: A community-based case control study. Indian J Comm Health. 2021;33(2):391-393. https://doi.org/10.47203/IJCH.2021.v33i02.030

Source of Funding: Tuberculosis Association of Telangana Conflict of Interest: None declared

\section{Article Cycle}

Received: 18/05/2021; Revision: 07/06/2021; Accepted:26/06/2021; Published:30/06/2021

This work is licensed under a Creative Commons Attribution 4.0 International License.

\section{Abstract}

Studies on risk factors of tuberculosis help to focus on risk of tuberculosis and take appropriate policy action which help to eliminate tuberculosis. Hence present study was carried out to study risk factors of pulmonary tuberculosis. Communitybased case-control studywas carried out among 78 age and sex matched cases and controls. Cases were incident cases of pulmonary tuberculosis aged 18-45 years of either sex. Data on housing condition was recorded with direct observation. Cases and controls were comparable for age, sex, presence of diabetes, HIV and immuno-compromised status. Mean body mass index was significantly low in cases as compared to controls (18.06 vs. 22.05; $\mathrm{p}<0.0001$ ). On binomial logistic regression odds of tuberculosis was $5.544(95 \% \mathrm{Cl}=2.513-12.232 ; \mathrm{P}=0.000)$ among those exposed to indoor air pollution after controlling for other factors. We conclude that exposure to indoor air pollution was significantly associated with risk of pulmonary tuberculosis

\section{Keywords}

Tuberculosis; Biomass; Air Pollution; Smoking

\section{Introduction}

Annually around 2 million die due to tuberculosis. Most of these are from non-industrialized countries. Majority studies have focused on active/smoking and risk of tuberculosis while fewer studies focused on exposure to indoor air pollution. (1)

$50 \%$ of global population uses solid fuel for their day to day living and more so from countries in Asia and Africa. To significantly tackle public health burden of tuberculosis in view of increasing number of smokers and exposed to indoor air pollution it is essential to address this issue on large scale. (2)

Even though there is association between indoor air pollution and tuberculosis, causality is lacking. A strong relation between the presence of indoor air pollution and the risk of tuberculosis was shown in one study from North India after controlling for age but not controlled for socioeconomic factors. (3)

In spite of effective National Tuberculosis Elimination program; many cases continue to occur and research is needed to focus on more risk factors to address problem and make program more effective. This may be due to the fact that even today, many people are having bad housing conditions and do not have access to the smokeless fuel.

\section{Aim \& Objectives}

To study risk factors of pulmonary tuberculosis with special reference to effect of exposure to indoor air pollution

\section{Material \& Methods}

Community based case control study was carried out over three months from 1st October to 31st December 2019.

The sample size was calculated using the OpenEpi software (4) for case-control studies. Considering the two sided confidence level (1-alpha) as $95 \%$; with $80 \%$ power, ratio of controls to cases as 1 , and least extreme odds ratio 2.5 , and expected proportion in controls as $40 \%$; sample size was 78 in each group of cases and controls. One tuberculosis units (TU) was selected randomly. Tuberculosis patient's data was obtained from DTCO, Medchal. From this, 78 TB patients were selected 
randomly as cases using computer generated table of random numbers. The controls were age and sex matched neighborhood of the TB patient.

\section{Selection protocol of the patients}

Dundigal TU: Total TB patients from June 2019 to August $2019=239$

Belonging to age group of $18-45$ years $=208$

$18-45$ years + new cases $=179$

18-45 years + new cases + pulmonary tuberculosis $=121$

78 Random numbers from these 121 were generated using online random number generator available from: https://stattrek.com/statistics/random-number-

generator

\section{Inclusion criteria:}

1. Age 18 to 45 years of either gender

2. Incident cases of pulmonary TB on treatment for not more than three months

3. Controls without signs and symptoms of TB and age and sex matched with neighborhood cases

\section{Exclusion criteria}

1. Known cases of asthma or other lung diseases

2. Previous history of exposure to active TB case in last five years

Institutional Ethics Committee permission was obtained. Permission from the District Tuberculosis Control Officer, Medchal was obtained. Written informed consent was obtained from all study participants. Case definition was newly diagnosed case of pulmonary TB on treatment for not $>3$ months. Age and sex matched 78 controls were selected from neighborhood houses of selected cases. TB registration number last digit of cases was taken to select control house. If last digit was $n$, then nth house to right of case house was visited. If there were two controls, then one was selected randomly. If there are no controls or eligible control did not give informed consent then $n+2$ house was taken.

Exposure to indoor air pollution: A person was supposed to be exposed to the indoor air pollution if he gave history of use of biomass fuel in the house either at present i.e. at the time of the survey or in the past for a significant period of time i.e. more than five years

The data was collected in the questionnaire devised by Central Pollution Control Board, Government of India. (5) Statistical analysis:Logistic regression analysis was carried out to control for effect of confounding factors using SPSS software. $P$ value less than 0.05 was taken as statistically significant.Multi-co linearity was tested using variance inflation factor. Nagelkerke R Squarewas 0.256 which meant that $25.6 \%$ of the variation in the outcome was explained by the variables of interest. Hosmer and Lemeshow test was statistically not found to be significant which meant that the model was fit to predict outcome and there were no misspecifications in final model. Predictive accuracy of null model was $50 \%$ which increased to $66.7 \%$ after variables were entered in model which meant that predictive accuracy of model increased.

\section{Results}

It was found that mean BMI was very low in tuberculosis patients compared to controls. This was statistically significant. (Table 1)

Only few variables were entered in final model of binary logistic regression. Risk of tuberculosis was 5.544 times more in those who were exposed to indoor air pollution compared to those who were not exposed and this was significant. Similarly those who belonged to lower socialclass were found to be 2.317 times more exposed to the risk of tuberculosis than those who belonged to upper class and this odds was significant. Other factors like separate kitchen, duration, type of house, overcrowding and cross ventilation were not statistically significant. (Table 2)

\section{Discussion}

We observed that mean BMI was significantly less in patients with tuberculosis compared to controls. This association is bidirectional. Underweight can lead to increased risk of tuberculosis. At the same time, tuberculosis is an important cause of loss of weight and hence the reduced BMI.

We found that the risk of tuberculosis was 5.5 times more in those who were exposed to indoor air pollution and 2.3 times more among those who belonged to lower social classes. Other housing factors like type of house, absence of cross ventilation and absence of separate kitchen were not found to be significantly associated with risk of tuberculosis. Mishra VK et al (6) reported that odds of indoor air pollution were 3.56 which reduced to 2.58 when controlled for other housing and environmental factors. Kan X et al (7) observed that $73.8 \%$ of the tuberculosis cases were exposed to the risk of indoor air pollution as they were using solid fuel compared to $72.5 \%$ among controls and this difference was not found to be statistically significant. Sumpter $C$ et al (8) carried out a systematic review on association between indoor air pollution and risk of tuberculosis. They included 13 studies. They found that the pooled OR was $1.3(95 \%$ $\mathrm{Cl}=1.04-1.62 ; \mathrm{p}=0.02$ ) which suggested strong association between indoor air pollution and risk of tuberculosis. Lin $\mathrm{HH}$ et al (9) also carried out a systematic review and metaanalysis and included 38 studies. They concluded that even though there is evidence of increased risk of tuberculosis due to passive smoking and indoor air pollution, the available evidence to support this association is weak and there is need to conduct large community based studies. Ozturk $A B$ et al (10) found from their study that odds of having tuberculosis was $1.6(95 \%$ $\mathrm{Cl}: 1.179-2.305, \mathrm{p}<0.003)$ among those who used coal or wood for heating purpose.

Thus overall there is conflicting evidence on association between exposure to indoor air pollution and risk of tuberculosis. Continuous exposure to indoor air pollution smoke definitely increases the risk of developing 
tuberculosis as can be proved by biological plausibility and we also found the same.

\section{Conclusion}

Mean BMI was significantly less in tuberculosis patients. Exposure to indoor air pollution, and belonging to lower social class were significant determinants of tuberculosis. Hence basic improvements in the standards of living of populations can reduce the burden of tuberculosis to a significant level where it ceases to be a public health problem.

\section{Limitation of the study}

Present study was case-control study and hence exposed to biases. There may be recall bias or limitation of recall on exposure to indoor air pollution. Hence housing conditions were recorded by direct observation. Interviewer bias may have been introduced as he was not blinded for cases and controls. Direct measurements of the air pollutants inside the house were not done.

\section{Relevance of the study}

With Government of India focus on tuberculosis elimination by 2025; all attempts and research is required to focus on risk factors of tuberculosis, so that measures can be taken to reduce incidence of tuberculosis.

\section{Acknowledgement}

We are thankful to "Tuberculosis Association of Telangana (TAT)" for funding study. TAT provides financial assistance to short term research projects on topics/fields which are in line to control the Tuberculosis every year by sending notifications on priority research areas.

\section{References}

1. World Health Organization. Tobacco or health: A global status report. Geneva: WHO; 1997

2. Maurya V, Vijayan VK, Shah A. Smoking and tuberculosis: an association overlooked. Int J Tuberc Lung Dis. 2002 Nov;6(11):94251. PMID: 12475139.

3. Gupta BN, Mathur N, Mahendra PN, Srivastava AK. A study of the household environmental risk factors pertaining to respiratory diseases. Energy Environ Rev 1997; 13:61-67

4. Dean AG, Sullivan KM, Soe MM. OpenEpi: Open Source Epidemiologic Statistics for Public Health, Version. www.OpenEpi.com, updated 2013/04/06, (accessed 2021/06/25)

5. Central Pollution Control Board, Ministry of Environment \& Forests, Govt. Of India. Indoor Air Pollution, Monitoring Guidelines. Questionnaire for Indoor Air Quality Diagnostic Protocol. Central Pollution Control Board, Ministry of Environment \& Forests, Govt. Of India. East Arjun Nagar, Delhi, India. 2014:15-17

6. Mishra VK, Retherford RD, Smith KR. Biomass cooking fuels and prevalence of tuberculosis in India. Int J Infect Dis. 1999;3(3):119129

7. Kan X, Chiang CY, Enarson DA, Chen W, Yang J, Chen G. Indoor solid fuel use and tuberculosis in China: a matched case-control study. BMC Public Health. 2011;11:498

8. Sumpter C, Chandramohan D. Systematic review and meta-analysis of the associations between indoor air pollution and tuberculosis. Trop Med Int Health. 2013;18(1):101-108

9. Lin HH, Ezzati M, Murray M. Tobacco smoke, indoor air pollution and tuberculosis: a systematic review and meta-analysis. PLoS Med. 2007;4(1):e20

10. Ozturk AB, Kilicaslan Z, Issever H. Effect of smoking and indoor air pollution on the risk of tuberculosis: smoking, indoor air pollution and tuberculosis. Tuberk Toraks. 2014;62(1):1-6

\section{Tables}

TABLE 1ASSOCIATION BETWEEN MEAN BMI \& TUBERCULOSIS

$\begin{array}{lllll}\text { Body mass index } & \text { Cases } & \text { Controls } & \text { T test } & p \text { value } \\ \text { Mean BMI }\left(\mathbf{k g} / \mathrm{m}^{\mathbf{2}}\right) & 18.06 \pm 4.2 & 22.05+4.04 & \mathbf{6 . 0 4 8 6} & <0.0001\end{array}$

\section{TABLE 2 BINARY LOGISTIC REGRESSION MODEL FOR DETERMINANTS OF TUBERCULOSIS}

\begin{tabular}{|c|c|c|c|}
\hline Factors & & Odds Ratio (95\% Cl) & p value \\
\hline \multirow[t]{2}{*}{ Exposure to indoor air pollution } & Absent & 1 & - \\
\hline & Present & $5.544(2.513-12.232)$ & 0.000 \\
\hline \multirow[t]{2}{*}{ Education } & Lower (illiterate + primary) & 1 & - \\
\hline & Higher (secondary + higher) & $1.035(0.491-2.182)$ & 0.929 \\
\hline \multirow[t]{2}{*}{ Socio-economic status } & Upper (class II + III) & 1 & - \\
\hline & Lower (class IV + V) & $2.317(1.137-4.720)$ & 0.021 \\
\hline \multirow[t]{2}{*}{ Type of house } & Pakka & 1 & - \\
\hline & Semi-pakka & $0.721(0.346-1.502)$ & 0.381 \\
\hline \multirow[t]{2}{*}{ Overcrowding } & No & 1 & - \\
\hline & Yes & $0.921(0.428-1.980)$ & 0.833 \\
\hline \multirow[t]{2}{*}{ Cross ventilation } & Yes & 1 & - \\
\hline & No & $1.348(0.493-3.686)$ & 0.561 \\
\hline \multirow[t]{2}{*}{ Separate kitchen } & Yes & 1 & - \\
\hline & No & $0.513(0.198-1.328)$ & 0.169 \\
\hline
\end{tabular}

